\title{
A New XRD Method to Quantify Plate and Lath Martensites of Hardened Medium-Carbon Steel
}

\author{
Quanshun Luo
}

\author{
(Submitted December 1, 2015; in revised form February 23, 2016; published online April 20, 2016)
}

\begin{abstract}
This paper introduces a new technique to separately measure the volume fraction and tetragonal ratio of co-existing lath and plate martensites in ultrahigh strength steel, and to calculate their different carbon contents. First, the two martensites are assumed to have body-centered tetragonal lattice structures of different tetragonal ratios. $X$-ray diffraction is then applied to obtain the overlapping $\{200\}$ diffraction peak, which is subsequently separated as four sub-peaks using a self-made multiple Gaussian peak-fitting method to allow the measurement of the individual lattice parameters $c$ and $a$. Finally, a modified equation is applied to calculate the carbon contents from the obtained tetragonal ratios. The new technique is then applied to investigate the effect of subsequent tempering on the decarbonization of the as-quenched martensites.
\end{abstract}

Keywords Gaussian peak-fitting, martensite carbon content, martensite tetragonal ratio, medium-carbon steels, Xray diffraction

\section{Introduction}

Carbon is an essential element in most ultrahigh strength steels, playing an important role both in strengthening mechanisms and in the transformation kinetics. Ultrahigh strength steels (UHSS) are key structural materials of aircrafts, automotive/rail vehicles, and advanced civil engineering structures (Ref 1,2). The latest development of ultrahigh strength steels has followed a new concept of microstructure design of multiphases, meta-stable, and multi-scales (Ref 3). In the last decade, a few novel heat treatment processes have emerged to enhance the strength and toughness properties, including quenchingpartitioning (Q-P) (Ref 4, 5), quenching-partitioning-tempering (Q-P-T) (Ref 6), and low-temperature bainitic isothermal treatment (Ref 7-9). However, it is still a technically challengeable issue to measure the carbon contents of martensitic or bainitic ferrites in most medium-carbon steels.

X-ray diffraction (XRD) analysis has been applied to determine the lattice parameters of martensitic ferrites by measuring the $\{200\}$ diffraction peak which, when the tetragonal ratio of the martensite is high enough, is well separated as two sub-peaks (200) and (002) (Ref 10, 11). This method, however, is not applicable to most hardened mediumcarbon steels. First, the microstructure of hardened mediumcarbon steel contains two types of martensite grains, namely the plate and lath martensites, having different carbon contents and thereafter different tetragonal ratios (Ref 12, 13). A successful measurement should include the two martensite types. Second, the resultant $\{200\}$ diffraction exhibits always as a single peak

Quanshun Luo, Materials and Engineering Research Institute, Sheffield Hallam University, Howard Street, Sheffield S1 1WB, UK. Contact e-mail: q.luo@shu.ac.uk.

because of severe peak overlapping. Up to now, XRD method described in Ref 10,11 is not applied to measure the martensites of medium-carbon steels because of the peak overlapping. Interestingly, a modified XRD analytical method was reported in Ref 14-16, which was applied to measure the carbon content of martensite in hardened medium-carbon steels. This method separately used the $\{200\}$ diffraction to measure parameter $\boldsymbol{a}$ and the $\{110\}$ diffraction to measure parameter $c$ of the tetragonal lattice. Here the martensite was obviously treated as a single phase with homogeneous carbon content. Similar to that, dilatometric technique is able to measure the starting temperature of martensite transformation, $M_{\mathrm{s}}$, and thereafter to estimate martensite carbon content (Ref 17). The dilatometric method again considers the martensite as a single phase, and fails to differentiate the carbon contents between martensite and retained austenite.

The carbon contents of austenite and martensitic or bainitic ferrites can also be measured by electron energy loss spectroscopy (EELS) (Ref 18, 19) and atomic probe tomography (APT) (Ref 5, 10, 20, 21). The advantages of these latest sophisticated analyses include their extremely high spatial resolution to determine the heterogeneous distributions of carbon at the bainite-austenite and martensite-austenite interfaces. However, such analyses require complicated sample preparation and are only available in a limited number of laboratories worldwide. Moreover, EELS analysis also suffers from carbon contamination in sample surface which makes quantitative analysis of carbon less reliable. In brief, there is no analytical technique available to provide quantitative characterization of co-existing lath and plate martensites of mediumcarbon steels.

In this paper, we introduce a new XRD method to determine the tetragonal ratios, and subsequently the different carbon contents, of the lath and plate martensites co-existing in hardened medium-carbon steel. The method was developed and extensively used in the recent research on novel heat treatments of ultrahigh strength steels having ultimate tensile strength $2100 \mathrm{MPa}$ and yielding strength $1750 \mathrm{MPa}$. The key step of the method is the separation of overlapping diffraction peaks using a self-made numerical process of Gaussian multiple peak- 
fitting. Then combining the conventional XRD analysis of retained austenite as described before, it has become feasible to quantify the volume fractions and carbon contents of the martensitic ferrites and retained austenite of ultrahigh strength steels.

\section{Sample Material, Characterization, and XRD Experiment}

The sample material being employed to demonstrate the method is a hot-rolled steel bar having chemical composition (in wt.\%) of C 0.55 , Ni 1.69, Cr 1.05, Mo 0.50, Mn 0.76, V 0.084 , and $\mathrm{Fe}$ in balance. The nominal $M_{\mathrm{s}}$ point (starting temperature of austenite-to-martensite transformation) of the sample steel is $230{ }^{\circ} \mathrm{C}$. Small rectangular samples of dimensions $20 \times 15 \times 8 \mathrm{~mm}$ were hardened by heating to an austenization temperature $850{ }^{\circ} \mathrm{C}$ and holding for $30 \mathrm{~min}$, oilquenching to room temperature (approximately $23{ }^{\circ} \mathrm{C}$ ), and tempering at temperatures of 200,250 , and $300{ }^{\circ} \mathrm{C}$ for $120 \mathrm{~min}$. The heat-treated samples were characterized by Vickers hardness, scanning electron microscopy (SEM), and transmission electron microscopy (TEM). A high-resolution FEG-SEM instrument, FEI NOVA 200, was employed for the SEM work using pre-polished and $2 \%$ nital pre-etched samples. A $200 \mathrm{kV}$ TEM instrument, Philips CM20 STEM, was used to characterize the martensite sub-structures. The TEM samples were first ground to $120-150 \mu \mathrm{m}$ thick, and then electrochemically polished in an electrolyte solution of $7 \%$ perchloric acid and 93\% glacial acetic acid at room temperature and a voltage of $32 \mathrm{~V}$. Some results of the microstructure characterization have been published in Ref 22.

A Philips X'Pert X-ray diffractometer with $\mathrm{Cu} \mathrm{K} \alpha$ radiation $(\lambda=0.154056 \mathrm{~nm}$, powered at $40 \mathrm{kV}$ and $40 \mathrm{~mA})$ was employed to acquire the $\{200\}_{M}$ and $\{211\}_{M}$ diffraction peaks of martensite and the $\{200\}_{\gamma},\{220\}_{\gamma}$, and $\{311\}_{\gamma}$ peaks of retained austenite, when the instrument was operated at the $\theta$ $2 \theta$ (Bragg-Brentano) mode. The diffraction peaks were obtained at a small step size of $0.0167^{\circ}$ and a long acquisition time of $1000 \mathrm{~s}$ per step. The as-acquired diffraction curve was processed by $\mathrm{K}_{\alpha 2}$ stripping and substrate removing.

This paper is focused on the quantification of the lath and plate martensites by proposing a new method to separate the $\{200\}_{M}$ diffraction peak. For the retained austenite, its volume fraction $(\gamma \%)$ was calculated using equation $\gamma \%=I_{\gamma} /\left(I_{\gamma} \pm G\right.$. $I_{\mathrm{M}}$ ), where $I_{\gamma}$ and $I_{\mathrm{M}}$ stand for the integrated intensities of the austenite and martensite diffraction peaks, respectively, and $G$ is a constant depending on the combination of the austenite and martensite planes. The values of the $G$ constants were adopted from selected literature (Ref 10, 11).

\section{The New Analytical Method to Separate Plate and Lath Martensites}

\subsection{Microstructure of the Oil-Quenched Steel}

Figure 1 shows the microstructure of the oil-quenched steel. Figure 1(a) is a SEM image showing the overall morphology of the as-quenched martensite. The martensite exhibits a complex mixture packet of parallel lath martensites and blocks of plate martensites as well as retained austenite. The morphology of lath martensites can be seen more clearly in Fig. 1(b), in which two blocks in the middle region are full of parallel martensite laths. Relatively large martensite plates locate adjacent to the lath-martensite blocks. The sub-structure of plate martensite is featured with twinning patterns, see Fig. 1(c) and (d). The twinning patterns evidence the shear-model mechanism of high-carbon martensite transformation. In particular, the TEM observations suggested co-existence of lath- and plate-type martensites which, as suggested in literature (Ref 12, 13), indicate heterogeneous carbon concentration, i.e., higher carbon content in plate-type martensites and lower carbon content in lath-type martensites.

To the best of the author's knowledge, most microscopic characterization of co-existing lath and plate martensites is qualitative. It is impossible to quantify co-existing lath and plate martensites from the SEM and TEM characterizations, because the lath and plate martensites, as shown in Fig. 1(a), are hardly differentiated to each other. Small observed area in each TEM image makes it impossible to undertake statistic measurements, although TEM does provide sufficient spatial resolution to show their different sub-structures. Another uncertainty of the quantification has been the difficult estimation of retained austenite which is either inter-lath filmy or block-like co-existing with the laths and plates, respectively.

Obviously, an alternative method needs to be developed for quantitative measurement. Figure 2 shows the diffraction peaks of the oil-quenched sample steel, including $\{200\}_{\mathrm{M}}$ and $\{211\}_{\mathrm{M}}$ of martensite and $\{200\}_{\gamma},\{220\}_{\gamma}$, and $\{311\}_{\gamma}$ of retained austenite. The five diffraction peaks have been used to determine the volume fraction and carbon content of retained austenite. Then the $\{200\}_{M}$ is to be used to discuss the new analytical method.

\subsection{Theoretical Basis for the Diffraction Peak Separation of Plate and Lath Martensites}

It is known that, martensite differs from ferrite in that most super-saturated carbon atoms in the former preferentially occupy the $[1 / 2,1 / 2,1]$ octagonal sites along the c-axis, as shown in Fig. 3. This contributes to a shift from body-centered cubic (bcc) lattice to body-centered tetragonal (bct) lattice with a tetragonal ratio $c / a>1$. In case of high-carbon martensite, such preferential distribution takes place through short-term diffusion of the carbon atoms soon after the austenite to martensite transformation to favor the lowest lattice strain energy (Ref 23-25). Because of the high-carbon concentration entrapped in the martensite lattice, the c/a ratio is large enough that, in a XRD pattern, the diffraction peaks $(200)_{M}$ and $(002)_{M}$ are well separated to each other to facilitate the measurement of the peak positions. Therefore, the determination of the tetragonal ratio $c / a$ is straight forward without any technical difficulty (Ref 10).

The martensite in hardened medium-carbon steel comprises a mixture of low-carbon martensitic laths and high-carbon martensitic plates (Ref 13). The kinetics of the lath and plate martensite formation has been well documented (Ref 12, 26, 27). Unlike high-carbon steels, the martensite transformation of medium-carbon steels comprises substantial carbon partitioning due to the relatively higher transformation temperature. As a result, carbon atoms entrapped in newly formed martensite laths have high mobility to diffuse into the adjacent austenite matrix to make the martensite depleting in carbon. Then the 

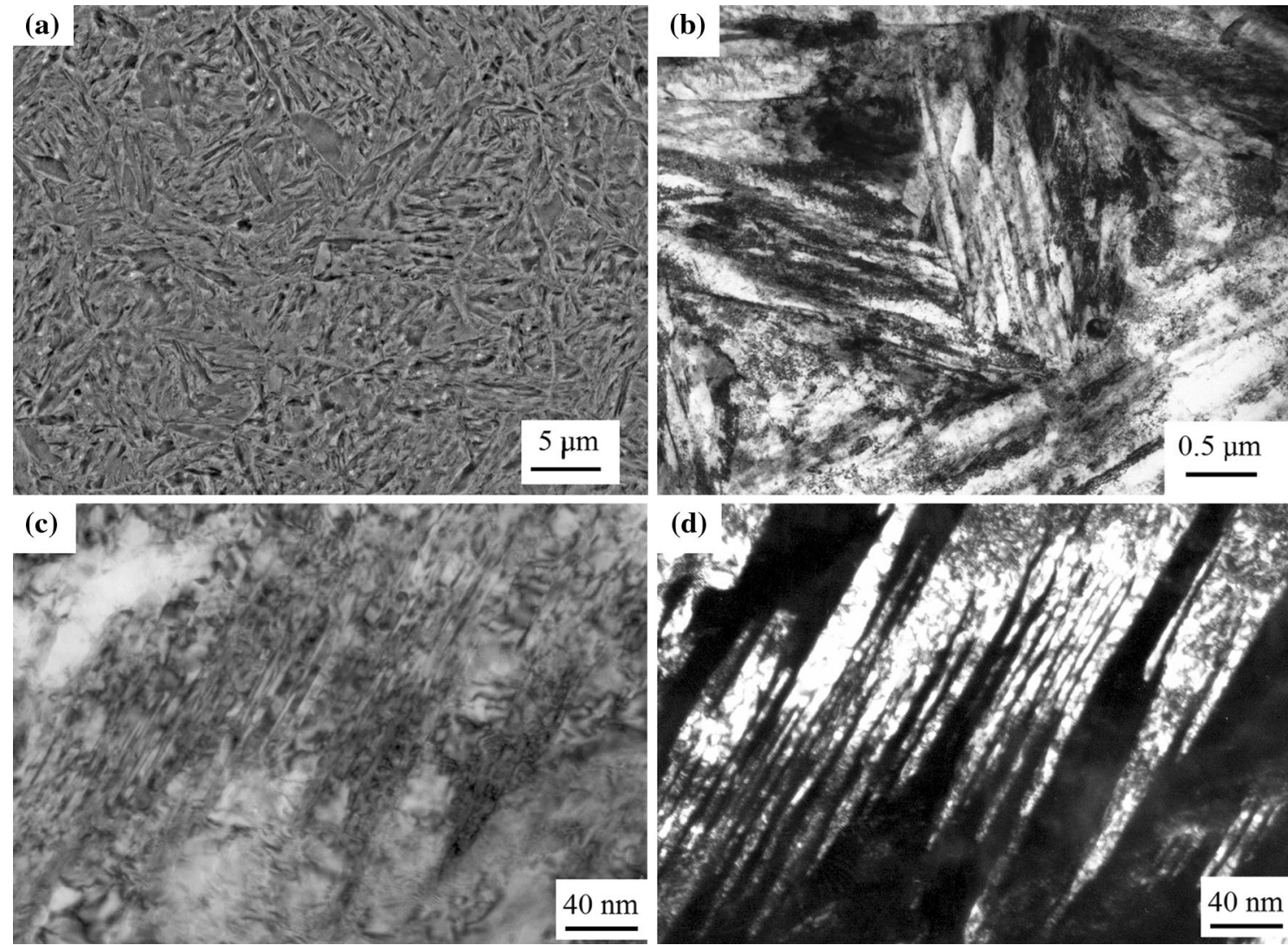

Fig. 1 Microstructure of the oil-quenched steel: (a) a scanning secondary electron image to show the overall martensite morphology; (b) a TEM bright-field image to show a few packets of lath martensites; (c) and (d) TEM bright-field and dark-field images of twinning sub-structure inside a plate-shaped martensite

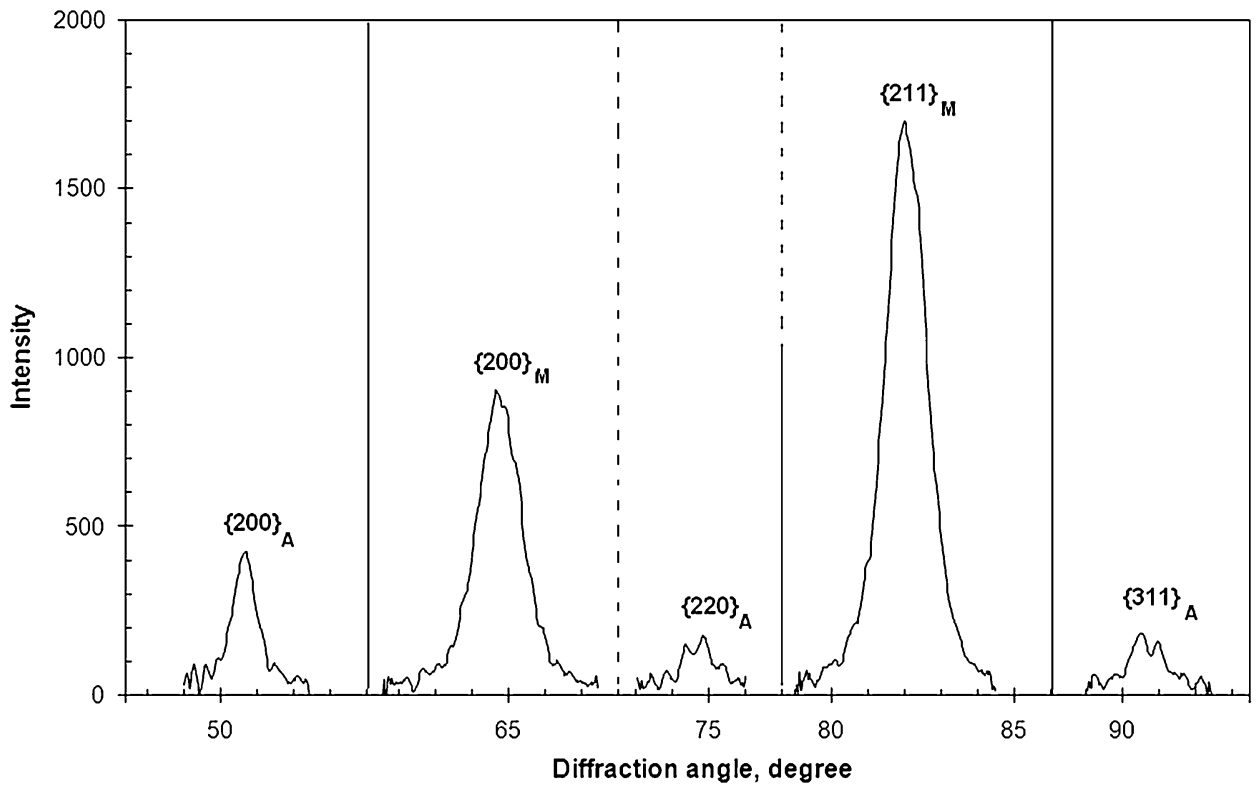

Fig. 2 X-ray diffraction peaks of the oil-quenched sample steel, including $\{200\}_{M}$ and $\{211\}_{M}$ of martensite and $\{200\}_{\gamma},\{220\}_{\gamma}$, and $\{311\}_{\gamma}$ of retained austenite

plate martensite formed at the lower temperature range remains higher carbon content due to the reduced carbon mobility. In brief, this results in heterogeneous distribution of carbon contents in the lath and plate martensites. The heterogeneous microstructure was confirmed in the quenched structures of a $0.12 \mathrm{C}$-low $\mathrm{Ni}-\mathrm{Cr}$ steel (Ref 12) and the samples of current study (Ref 22). 


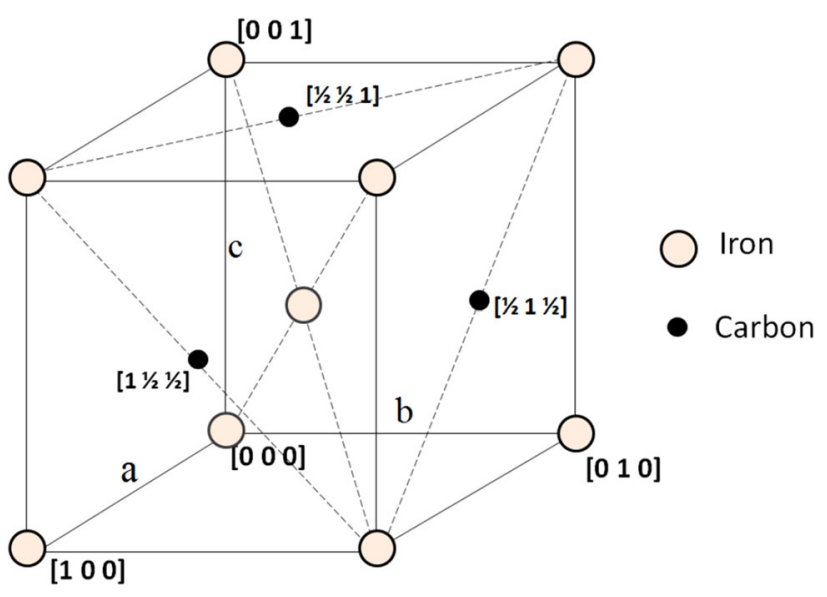

Fig. 3 A lattice cell of body-centered tetragonal martensite, where carbon occupies preferentially one of the three octagonal sites, i.e., the $[1 / 2,1 / 2,1]$ cite, leading to a tetragonal ratio $c / a>1$

Provided that the co-existing lath and plate martensites in a hardened medium-carbon steel have different carbon contents, they can be treated as two bct sub-phases having different tetragonal ratios. Then the overall $\{200\}_{\mathrm{M}}$ diffraction peak of martensite should comprise four sub-peaks, namely the $(200)_{M}$ and $(002)_{M}$ sub-peaks of the plate and lath martensites. However, retrieving of the overall $\{200\}_{M}$ diffraction peak has been only theoretically possible because of strong overlapping of the four sub-peaks. The overlapping is attributed to both the high similarity in the tetragonal ratios of the two martensitic sub-phases and the strong peak broadening arising from the severe lattice straining.

\subsection{Separation of Plate and Lath Martensites Using the Gaussian Peak-Fitting}

In this paper, a multi-peak Gaussian peak-fitting method is proposed to separate the four overlapping sub-peaks by following the following procedure.

(1) Setting up a Gaussian profile is a MS Excel file;

(2) Converting the pre-treated overall $\{200\}_{M}$ diffraction peak to a MS Excel file;

(3) Using two Gaussian profiles to fit the (200) plate and $(002)_{\text {plate }}$ sub-peaks of plate martensite in the overall $\{200\}_{M}$ diffraction peak;

(4) Subtracting the two Gaussian-fitted (200) plate and $(002)_{\text {plate }}$ sub-peaks from the overall $\{200\}_{M}$ peak, to get a remained peak in the middle position of the overall peak;

(5) Raising the third and fourth Gaussian profiles to fit the remaining peak as the $(200)_{\text {lath }}$ and $(002)_{\text {lath }}$ sub-peaks of the lath martensite;

(6) Optimizing the parameters of the four Gaussian profiles to make their sum intensity best fit to the overall $\{200\}_{M}$ diffraction peak;

(7) The obtained Gaussian profiles represent the four diffraction sub-peaks of the plate and lath martensites. For each profile, the peak position, integrated intensity and peak broadening (full-width at half-maximum) can be determined for further characterization of the martensites.
3.3.1 Setting up a Gaussian Profile to Fit to a Single Diffraction Peak. In the probability theory, Gaussian profile is a continuous probability distribution. It has been widely accepted that a single diffraction peak of a polycrystalline phase obeys the Gaussian profile (Ref 10, 28, 29). A modified Gaussian formula is shown in Eq 1 to express the diffraction intensity $(I)$ as a function of diffraction angle $(2 \theta)$. The shape and position of a Gaussian profile is determined by three independent constants $I_{0}, \mu$, and $\sigma$, where $I_{0}$ is a linear intensity parameter, $\mu$ is the mean or expectation of the original Gaussian distribution and here it determines the position of the curve with respect to diffraction angle $2 \theta$, and $\sigma$ is the standard deviation in the original Gaussian distribution and here it determines the scale of peak broadening.

$I_{(2 \theta)}=\frac{I_{0}}{\sigma \sqrt{2 \pi}} \cdot e^{-\frac{(2 \theta-\mu)^{2}}{2 \sigma^{2}}}$.

As shown in Eq 1, a Gaussian profile is determined by setting the values of a group of $I_{0}, \mu$, and $\sigma$ parameters. Thus, such a profile can be easily set up in a MS Excel data sheet to fit to a single diffraction peak.

3.3.2 Raising Two Gaussian Profiles to Fit the (200) $)_{\text {plate }}$ and (002) $)_{\text {plate }}$ Sub-peaks. The multiple peak-fitting process is illustrated in Fig. 4. The $\{200\}_{M}$ diffraction is shown in Fig. 4(a) in higher precision than those in Fig. 2. For an overlapping diffraction peak containing two diffraction subpeaks, a MS-Excel-based Gaussian peak-fitting technique has been demonstrated to be successful in separating overlapping diffraction peaks of gamma prime precipitate and the parent gamma phase of a long-term aged Nimonic 263 superalloy (Ref $30)$. In the current work, two Gaussian profiles are generated to represent the diffraction peaks $(200)_{\text {plate }}$ and $(002)_{\text {plate }}$ of plate martensite. Several principles should be followed in the peak fitting.

First, the $(200)_{\text {plate }}$ and $(002)_{\text {plate }}$ peaks should fit to the lower and broader part of the overall $\{200\}_{\mathrm{M}}$ peak. This is because the plate martensite has higher carbon content and consequently larger tetragonal ratio than the co-existing lath martensite. Meanwhile, the higher carbon content of the plate martensite also contributes to its greater lattice strain. Second, the $(002)_{\text {plate }}$ peak is allocated on the left-hand side and the $(200)_{\text {plate }}$ peak on the right-hand side, given the tetragonal ratio $c / a>1$.

Then two simplifying assumptions are made in the peakfitting. First, the Gaussian profiles, (200) $)_{\text {plate }}$ and (002) $)_{\text {plate, }}$ are assumed to have an identical $\sigma$ value, for the $\sigma$ value scales with the peak broadening, or the lattice straining, of the same martensite constituent. Second, the intensity parameter $I_{0}$ of the $(200)_{\text {plate }}$ peak should be double that of the $(002)_{\text {plate }}$ peak, for the former include diffractions from the (200) and (020) planes and assuming the sample to be analyzed is polycrystalline with random orientation.

Consequently, the two Gaussian profiles should have only four independent variables, namely the same $I_{0}$ and $\sigma$ parameters for the $(200)_{\text {plate }}$ and $(002)_{\text {plate }}$ peaks, and two different $\mu$ values for the two peaks. By adjusting the four variables, the two sub-peaks are fitted to the edges of the bottom part of the overall $\{200\}_{M}$ peak, as shown in Fig. 4(b).

3.3.3 Raising the Third and Fourth Gaussian Profiles to Fit the (200) lath and (002) lath Sub-peaks. The remaining intensity profile, peak ' 4 ' in Fig. 4(b), should be a sum of the 

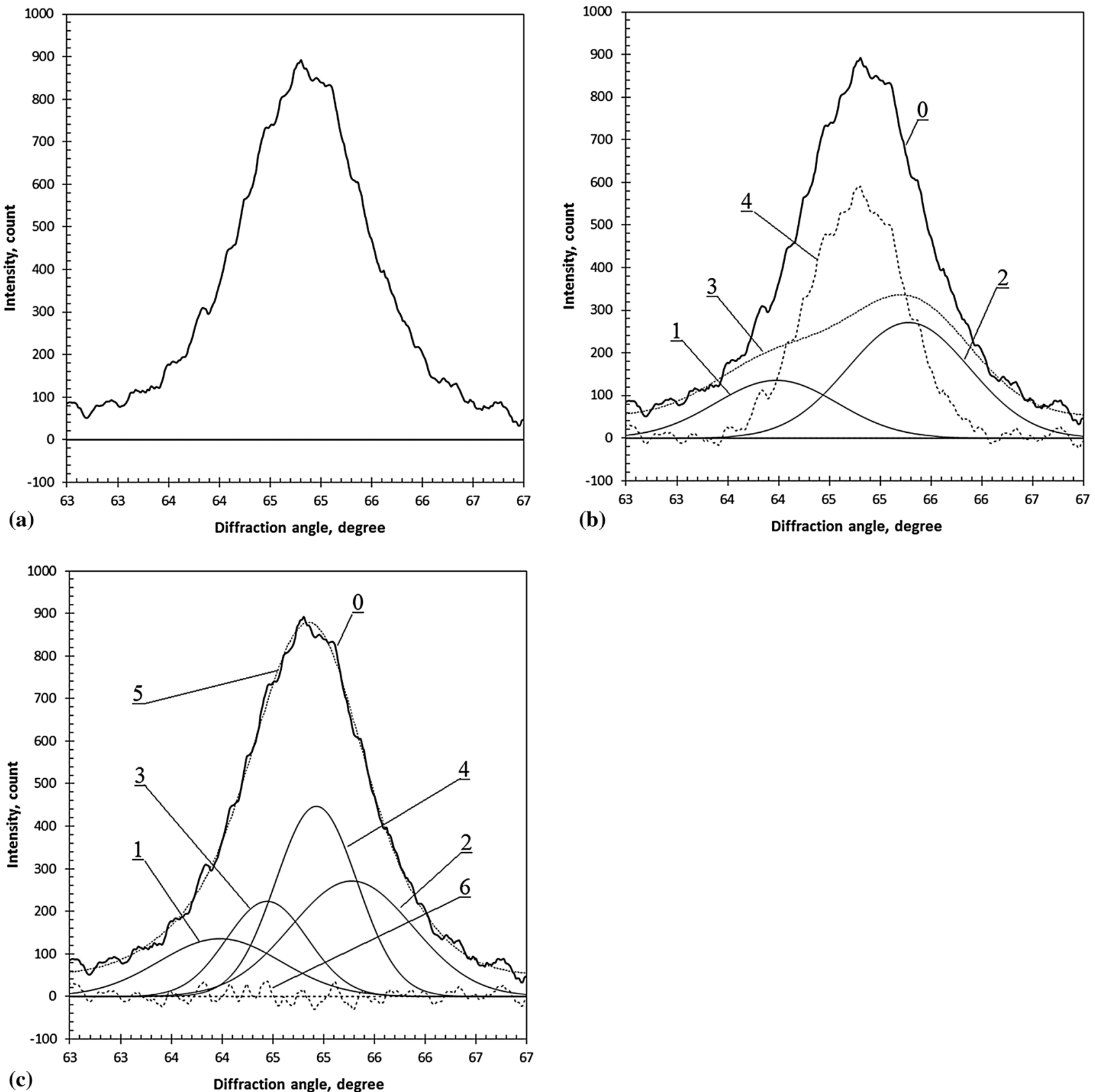

Fig. 4 Multiple Gaussian peak-fitting of the $\{200\}_{M}$ diffraction. (a) The $\{200\}_{M}$ diffraction peak of the oil-quenched sample steel. (b) Two Gaussian profiles to fit to the root part of the $\{200\}$ diffraction peak: peaks ' 1 ' and ' 2 ' are Gaussian profiles to present the $(002)_{\text {plate }}$ and (200) plate, respectively; profile ' 3 ' is the sum of the two Gaussian profiles; peak ' 0 ' is the as-measured $\{200\}$ diffraction and peak ' 4 ' is the remaining intensity profile. (c) Completion of the multiple Gaussian peak fitting: peaks 1-4 represent the $(002)_{\text {plate }},(200)_{\text {plate }},(002)_{\text {lath }}$, and (200) lath sub-peaks, respectively; peak 5 is their sum intensity; peak ' 0 ' is the as-measured $\{200\}$ diffraction and peak 6 is the difference between peaks 0 and 5

two diffraction sub-peaks of the lath martensite, namely the $(200)_{\text {lath }}$ and $(020)_{\text {lath }}$ peaks. Then, the third and fourth Gaussian profiles are generated to fit the rest intensity profile by following the same principles as describe above.

After the fitting, ideally the total integrated intensity of the four Gaussian profiles generated above should match to the overall $\{200\}_{M}$ peak. However, further improvement can be made by more precisely adjusting the $I_{0}, \mu$, and $\sigma$ parameters of the four profiles, to minimize the fitting error, i.e., the difference between the sum of the four profiles and the integrated intensity of the overall $\{200\}_{M}$ peak. The resultant fittings are shown in Fig. 4(c) and resultant parameters of the four Gaussian profiles are given in Table 1.

In Table 1 , the $\mu$ values refer to the diffraction angles $2 \theta$, from which the lattice parameters $\boldsymbol{a}$ and $\boldsymbol{c}$ as well as the tetragonal ratio $c / a$ are determined. The $\sigma$ parameter refers to the peak broadening of the two martensites. As expected, the plate martensite shows greater peak broadening than the lath martensite. The $I_{\text {sum }}$ parameter refers to integrated intensity of each Gaussian profile, which is proportional to the volume 
Table 1 Parameters of the Gaussian profiles referring to the plate and lath martensites

\begin{tabular}{|c|c|c|c|c|}
\hline & $(200)_{\text {plate }}$ & $(002)_{\text {plate }}$ & $(200)_{\text {lath }}$ & $(002)_{\text {lath }}$ \\
\hline$\mu, \operatorname{deg}$ & 65.28 & 63.98 & 64.93 & 64.44 \\
\hline$a, c, \mathrm{~nm}$ & 0.2856 & 0.2908 & 0.2870 & 0.2889 \\
\hline$c / a$ ratio & 1.0181 & & 1.0067 & \\
\hline$\sigma$ & 0.6 & 0.6 & 0.4 & 0.4 \\
\hline$I_{\text {sum }}$ & 23,997 & 12,000 & 26,353 & 13,176 \\
\hline
\end{tabular}

Table 2 Effect of tempering temperature on the hardness property

\begin{tabular}{lllll}
\hline No. & Austenization & Cooling & Tempering & HV \\
\hline 1 & $850^{\circ} \mathrm{C} / 25 \mathrm{~min}$ & Oil & $\mathrm{n} / \mathrm{a}$ & $720 \pm 27$ \\
2 & $850^{\circ} \mathrm{C} / 25 \mathrm{~min}$ & Oil & $200^{\circ} \mathrm{C} / 120 \mathrm{~min}$ & $677 \pm 17$ \\
3 & $850^{\circ} \mathrm{C} / 25 \mathrm{~min}$ & Oil & $250{ }^{\circ} \mathrm{C} / 120 \mathrm{~min}$ & $626 \pm 17$ \\
4 & $850^{\circ} \mathrm{C} / 25 \mathrm{~min}$ & Oil & $300^{\circ} \mathrm{C} / 120 \mathrm{~min}$ & \\
5 & $850^{\circ} \mathrm{C} / 25 \mathrm{~min}$ & Oil & $350{ }^{\circ} \mathrm{C} / 120 \mathrm{~min}$ & \\
6 & $850^{\circ} \mathrm{C} / 25 \mathrm{~min}$ & Oil & $400^{\circ} \mathrm{C} / 120 \mathrm{~min}$ & $568 \pm 5$ \\
\hline
\end{tabular}

fractions of the two martensites, from which a ratio can be determined to be $M_{\text {plate }}: M_{\text {lath }}=47.7: 52.3$.

\subsection{Calculating the Carbon Contents of Lath and Plate Martensites}

Previous research on hardened high-carbon steels revealed statistically linear relations between the martensite carbon contents and the lattice parameters $\boldsymbol{a}$ and $\boldsymbol{c}(\operatorname{Ref} 11,15,23)$. Equation 2 expresses the linear relations, where $a_{0}$ stands for the lattice parameter of pure $\alpha-\mathrm{Fe}, C$ stands for the carbon content (in wt.\%) of martensite, and the constants $A$ and $B$ derive from linear regressions of experiment data. This paper has adopted the values of $A=0.00124, B=0.0115$, and $\boldsymbol{a}_{\mathbf{0}}=0.28674 \mathrm{~nm}(\operatorname{Ref} 11)$. Then the two equations are rewritten as Eq 3. Meanwhile, a simple mathematical treatment of the two equations turns out a new equation to show the relation between carbon content $C$ and the tetragonal ratio $c / a$ as shown in Eq 4. The advantage of $\mathrm{Eq} 4$ is that both parameters $\boldsymbol{a}$ and $\boldsymbol{c}$ are involved in the calculation and each $\boldsymbol{c} / \boldsymbol{a}$ ratio works out to a single $C$ value

$a=a_{0}-A \cdot C ; \quad c=a_{0}+B \cdot C$

$C=231.24-806.45 \cdot a ; \quad C=24.93 \cdot c-86.96$

$C=\frac{0.28674\left(\frac{c}{a}-1\right)}{0.0115+0.00124 \frac{c}{a}}$.

If Eq 4 is used in a hardened medium-carbon steel, however, the carbon content would be significantly under-estimated, because it takes account only the carbon atoms which contribute to the tetragoneity by occupying the $[0,0,1 / 2]$ octahedral sites. It has been stated in the literature that not all the carbon atoms occupy the $[0,0,1 / 2]$ octahedral sites in a martensitic ferrite. Instead, there are always some carbon atoms which either exhibit random occupation among the three types of octahedral sites or preferentially sink along dislocations, and therefore do not contribute to the lattice tetragoneity. Such cubic structure could accommodate carbon atoms for up to $0.18 \%$ without changing to tetragonal structure (Ref 15, 16,

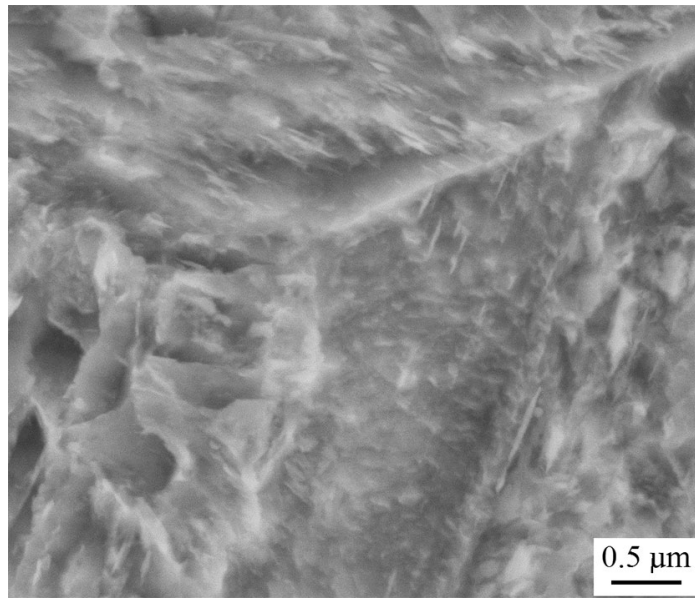

Fig. 5 A SEM micrograph showing carbide precipitates in the martensitic grains of $300^{\circ} \mathrm{C}$ tempered sample

23). More recently, APT analyses of bainitic and martensitic ferrites have confirmed enriched distribution of carbon along dislocations (Ref 21). Thus, a modified equation is proposed to include the randomly distributing carbon, in Eq 5

$C=\frac{0.28674\left(\frac{c}{a}-1\right)}{0.0115+0.00124 \frac{c}{a}}+0.18$.

Then using the $\boldsymbol{c} / \boldsymbol{a}$ values listed in Table 1, the carbon contents of the plate and lath martensites are calculated to be 0.687 and $0.367 \%$, respectively. Considering the relative fraction of the two martensites, the overall carbon content of the martensite matrix is estimated to be $0.52 \%$. This value has been slightly lower than the average carbon content of the steel $(0.55 \%)$ as a result of carbon partitioning during the austenite to martensite transformation. Previously, we have measured the volume fraction and carbon content of the retained austenite to be 8.0 and $0.90 \%$, respectively (Ref 22). By combining the measurements on the three microstructure 

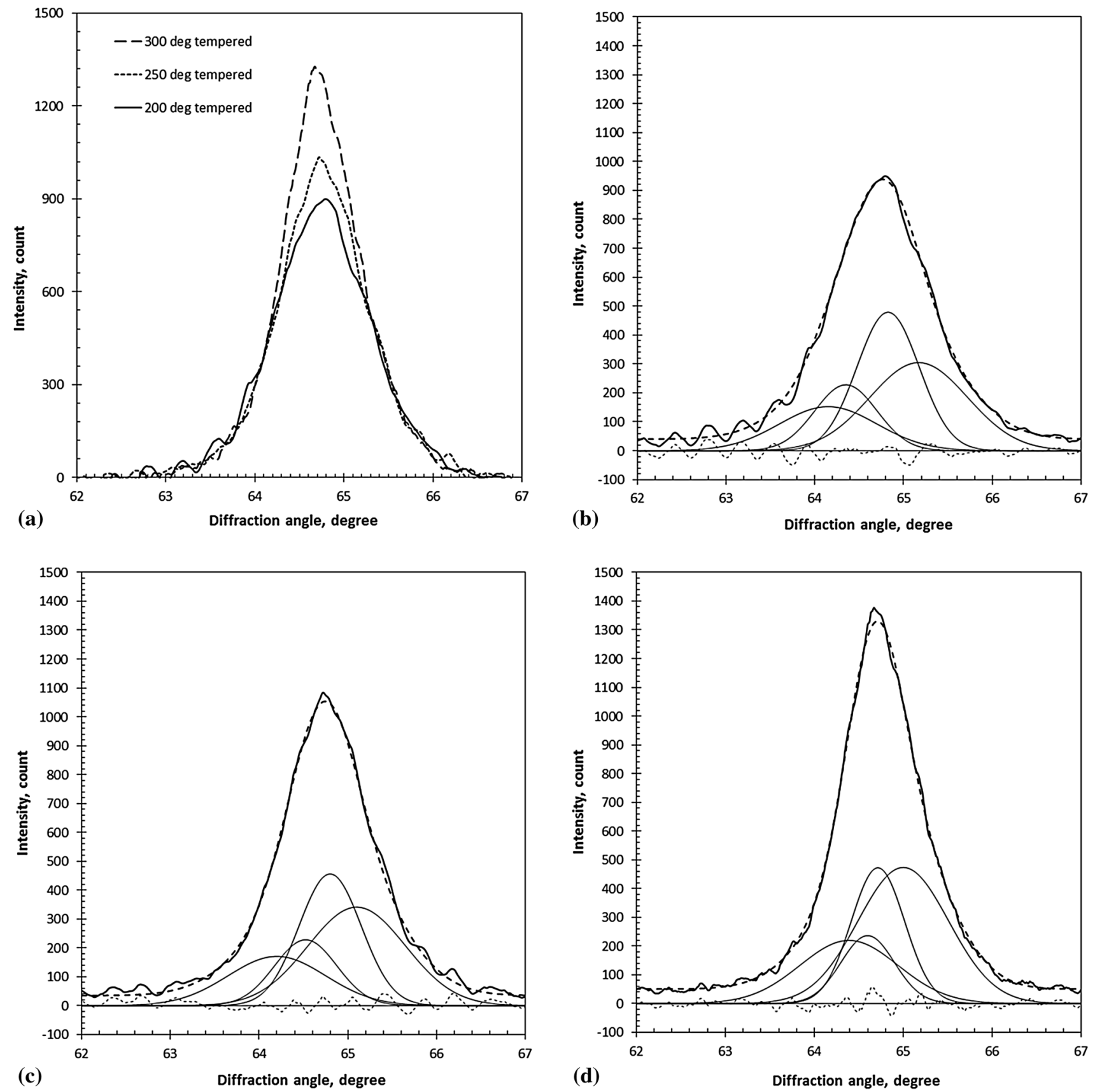

Fig. 6 Multiple Gaussian peak-fitting charts of the samples being tempered at various temperatures: (a) comparison between the $\{200\}_{M}$ diffraction peaks, noting the decreased peak broadening with increasing tempering temperature; (b) the $\{200\}_{M}$ diffraction peak of the sample tempered at $200{ }^{\circ} \mathrm{C}$; (c) the $\{200\}_{\mathrm{M}}$ diffraction peak of the sample tempered at $250{ }^{\circ} \mathrm{C}$; and (d) the $\{200\}_{\mathrm{M}}$ diffraction peak of the sample tempered at $300{ }^{\circ} \mathrm{C}$

constituents, the overall carbon content of the hardened matrix is $0.55 \%$, which matches well to the nominal carbon content of the steel.

\subsection{An Example of Applications: Decarbonization of Martensite in Tempering Treatment}

The new XRD method has been applied to investigate the effect of tempering treatment on the decarbonization of martensite. When the oil-quenched steel was tempered at a temperature from 200 to $400{ }^{\circ} \mathrm{C}$, it exhibited substantial decrease in hardness with the increasing tempering temperature, as shown in Table 2. SEM observations found plenty of fine carbide precipitates inside the tempered martensite grains. An example is shown in Fig. 5. The carbide precipitation is expected to lead to decarbonization of the martensitic ferrite substrate. The newly developed XRD method has been applied to analyze the decarbonization, as shown in Fig. 6. In Fig. 6(a), the $\{200\}_{M}$ peaks exhibit decreased broadening with increased tempering temperature. In Fig. 6(b)-(d), each $\{200\}_{\mathrm{M}}$ peak has been separated as four sub-peaks by following the procedure as described 


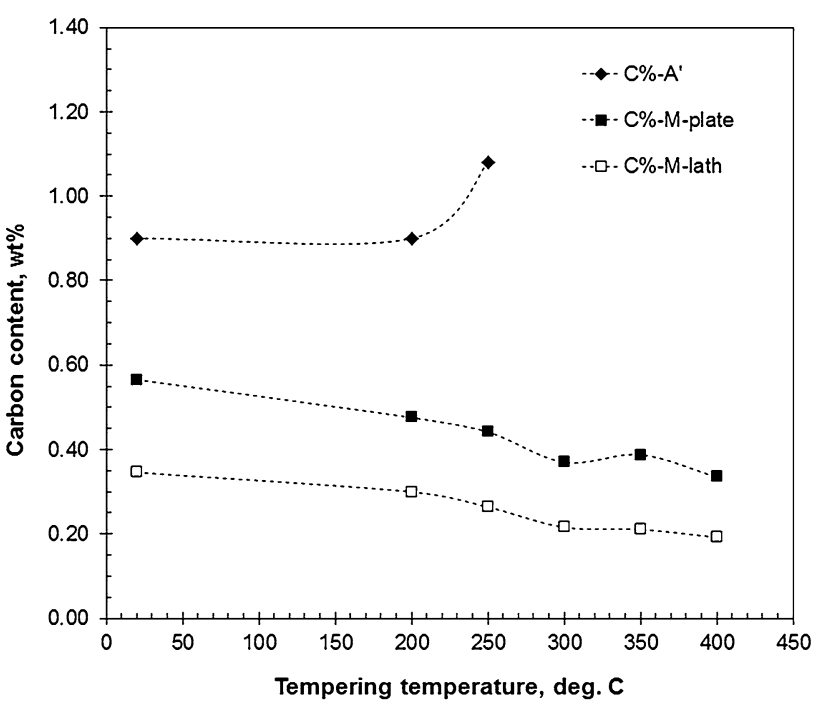

Fig. 7 Variation of the carbon contents of martensites and retained austenite with increasing tempering temperature

above. Consequently, the lath and plate martensites of the tempered samples have been quantified. Figure 7 shows carbon contents of the lath and plate martensites as well as retained austenite as determined from the XRD analyses as a function of the tempering temperature. The results confirm decarbonization of the tempered martensites. Moreover, the retained austenite exhibits substantially high-carbon content than the martensites. To the author's knowledge, it is the first time that the carbon concentration of the three structural constituents of hardened medium-carbon steel can be measured effectively using XRD techniques.

\section{Discussion}

This paper has introduced an XRD method to analyze the martensite structure of hardened medium-carbon steels. The method is based on two principal considerations. First, based on the tetragonal crystalline structure of martensite, it proposes a multiple Gaussian peak-fitting technique to retrieve the overall $\{200\}_{M}$ diffraction peak as four individual sub-peaks of the (200) and (002) peaks of lath and plate martensites. Consequently, the tetragonal ratio and relative fraction of the two martensites can be calculated, Fig. 4. Second, this approach has considered both carbon atoms contributing to the tetragoneity, owing to their preferential distribution, and those not contributing to the tetragoneity. Based on that, a modified equation has been proposed to calculate the carbon concentration of martensite from its tetragonal ratio, Eq 5. Thus, combining with the existing XRD method of retained austenite measurement, it is possible to provide quantitative measurement of the volume fraction and carbon concentration of the three structural constituents of hardened medium-carbon steels, namely the lath and plate martensites and retained austenite.

It is believed that the new method would help improve characterization of hardened steels and contribute to the development of new strengthening and toughening heat treatments. The research on martensitic structures have been enormous, which however concerned mostly the morphology and crystallographic characteristics but little on their carbon contents (Ref 13, 23-25, 31, 32). On the other hand, it is appreciated that comprehensive fundamental research on the carbon partitioning behavior in the martensitic and bainitic transformation of under-cooled austenite gave birth to several novel strengthening processes, including quenching-partitioning (Ref 4, 5), quenching-partitioning-tempering (Ref 6), and superbainitic isothermal treatments (Ref 7-9). The new XRD method would provide further opportunity to characterize the co-existing lath and plate martensites for their different carbon enrichment, as well as to investigate the structural evolution of hardened steels in subsequent tempering. In fact, it has played an important role in recent research of ultrahigh strength steels, whereas some example analyses have been given in this paper, see section 3.5, as well as in other recent papers ( Ref 22, 33). In particular, the newly developed quantitative analysis of coexisting lath and plate martensites provide a complementary tool to the existing analytical techniques, such as field emission SEM and TEM (Fig. 1 and 5), electron back-scattered diffraction (known as EBSD) (Ref 34, 35), APT (Ref 20, 21), and TEM-EELS (Ref 18, 19).

The relationship between the lattice parameters $a$ and $c$ of tetragonal martensite and its carbon content was derived from XRD experiment results of quenched high-carbon steels (Ref 23 ), and has been widely adopted as the basis in the estimation of martensite carbon contents (Ref 10, 11, 14-16). XRD and Gaussian peak-fitting techniques have been employed in quantitative analysis of high-carbon martensites for many years (Ref $10,11,14-16)$. This paper differs from those that, for the first time, it extends the quantitative analysis to hardened medium-carbon steels. Meanwhile, the author has adopted the opinion of Liu and co-workers (Ref 15,16$)$ that in a tetragonal martensite structure there are always some carbon atoms which do not contribute to the lattice tetragoneity through either randomly occupying some octagonal vacancies or agglomerating along dislocations. The linear relations (Eq 2 and 3) could only determine the concentration of carbon which preferentially occupies the $[1 / 2,1 / 2,1]$ vacancies, Fig. 3. Therefore, unlike the approximation proposed in literature (Ref 14-16), we assume that there is a minimum amount of carbon atoms which exhibits randomly in the tetragonal lattice making no contribution to the tetragonal ratio. The actual carbon content should include both the randomly and preferentially distributed parts of carbon, Eq 5. This assumption is consistent to the experimental and theoretical results of literature (Ref 15, 16, 25).

It should be pointed out that, uncertainty may exist when the carbon content is calculated from the tetragonal ratio of a martensite using Eq 5, where the maximum concentration of randomly distributing carbon has been estimated to be $0.18 \%$. In fact, the distribution of carbon atoms in a ferrite lattice depends strongly on the type and quantity of alloying elements. For example, it is known that, the out shell electrons of different alloying elements have different repulsive or attractive interactions with the out shell electrons of carbon atoms (Ref 36). These interactions determine the chemical bonds between carbon and the different metal elements and consequently influence the carbon distribution. Obviously, it is not the aim of this paper to make further discussion on this issue, whereas there is still lack of theoretical understanding on the occupation statue of carbon in a martensite structure (Ref $23-25,31,32$ ). Nevertheless, by assuming $0.18 \%$ of randomly distributed carbon, the estimated carbon contents of the martensites agree well to the actual composition of the sample steel. 
The limitation of this method is that it cannot be applied to measure the carbon content of a martensitic ferrite if their carbon content is less than $0.18 \%$ and therefore exhibits no measurable tetragoneity. Such low-carbon ferrites do exist, e.g., in some isothermal treated bainitic steels and quenched lowcarbon steels (Ref 7, 14, 16, 32).

\section{Summary}

A new XRD technique has been described in this paper which provides accurate measurement of the tetragonal ratios and volume fractions of lath and plate martensites co-existing in the quenched structure of a medium-carbon alloy steel.

(1) By assuming the same bct lattice structure and different lattice parameters of the two martensites, a Gaussian peak-fitting method has been applied to separate the overlapping diffraction peak $\{200\}$ as four sub-peaks representing the (200) and (002) diffractions of the two martensites.

(2) Using the obtained tetragonal ratios, a modified equation has been proposed to calculate the carbon contents with an assumption that a cubic ferrite phase can accommodate randomly distributed carbon up to $0.18 \%$ without changing to a tetragonal phase. The calculated carbon contents have been confirmed to match well to the actual composition of the sample steel.

(3) As an example, the new technique has been applied to measure the tetragonal ratios and carbon contents of lath and plate martensites of the hardened steel, which shows that the carbon contents of both martensites decrease with increasing tempering temperature.

\section{Acknowledgments}

The author acknowledges the partial financial support of Innovate UK (formerly Technology Strategy Board of the UK government, Smart Award No. 720113). The sample steel was provided by Tinsley Bridge Ltd through the Smart Award Project.

\section{Open Access}

This article is distributed under the terms of the Creative Commons Attribution 4.0 International License (http://creativecommons.org/ licenses/by/4.0/), which permits unrestricted use, distribution, and reproduction in any medium, provided you give appropriate credit to the original author(s) and the source, provide a link to the Creative Commons license, and indicate if changes were made.

\section{References}

1. Z. Li, Z.Q. He, J.J. Jin, and P. Zhong, Development of Aeronautical Ultra-High Strength Steels, National Defence Industry Press, Beijing, 2012, p 227 (in Chinese)

2. Y. Tomita, Development of Fracture Toughness of Ultrahigh Strength, Medium Carbon, Low Alloy Steels for Aerospace Applications, Int. Mater. Rev., 2000, 45, p 27-37

3. H. Dong, High Performance Steels: Initiative and Practice, Sci. China Technol. Sci., 2012, 55, p 1774-1790
4. D.V. Edmonds, K. He, F.C. Rizzo, B.C. De Cooman, D.K. Matlock, and J.G. Speer, Quenching and Partitioning Martensite-A Novel Steel Heat Treatment, Mater. Sci. Eng., 2006, A438-440, p 25-34

5. J. Speer, D.K. Matlock, B.C. DeCooman, and J.G. Schroth, Carbon Partitioning into Austenite After Martensite Transformation, Acta Mater, 2003, 51, p 2611-2622

6. Y. Rong, Advanced Q-P-T Steels with Ultrahigh Strength-High Ductility, Acta Metall. Sin., 2011, 47, p 1483-1489 (in Chinese)

7. F.G. Caballero and H.K.D.H. Bhadeshia, Very Strong Bainite, Curr. Opin. Solid State Mater, 2004, 8, p 251-257

8. T.S. Wang, J. Yang, C.J. Shang, X.Y. Li, B. Zhang, and F.C. Zhang, Microstructure and Impact Toughness of Low-Alloy High-Carbon Steel Austempered at Low Temperature, Scr. Mater., 2009, 61, p 434 437

9. C. Garcia-Mateo and F.G. Caballero, Ultra High Strength Bainitic Steels, ISIJ Int., 2005, 45, p 1736-1740

10. A. Garg and T.R. McNelley, Estimation of Martensite Carbon Content in As-Quenched AISI, 52100 Steel by X-ray Diffraction, Mater. Lett., 1986, 4, p 214-218

11. R. Abbaschian and L. Abbeschian, Physical Metallurgy Principles, 4th ed., Cengage Learning, Stanford, 1994, p 623-624

12. T.Y. Hsu and X.M. Li, Diffusion of Carbon During the Formation of Low-Carbon Martensite, Scr. Metall., 1983, 19, p A83-A88

13. G. Krauss, Deformation and Fracture in Martensitic Carbon Steels Tempered at Low Temperatures, Metall. Mater. Trans. A, 2001, 32, p 205-221

14. M.K. Kang, Y.L. Ai, M.X. Zhang, Y.Q. Yang, M. Zhu, and Y. Chen, Carbon Content of Bainite Ferrite in 40CrMnSiMoV Steel, Mater. Chem. Phys., 2009, 118, p 438-441

15. X. Liu, F. Zhong, J.X. Zhang, M.X. Zhang, M.K. Kang, and Z.Q. Guo, Lattice-Parameter Variation with Carbon Content of Martensite. 1. Xray Diffraction Experimental Study, Phys. Rev. B, 1995, 52, p 9970 9978

16. X. Liu and K.M. Kang, Quantitative Relationship Between the Lattice Parameters and Carbon Content of Martensite: New Experiments of Xray Diffraction, Trans. Met. Heat Treat., 2000, 21, p 68-77 (in Chinese)

17. Z. Lawrynowicz, Carbon Partitioning During Bainite Transformation in Low Alloy Steels, Mater. Sci. Technol., 2002, 18, p 1322-1324

18. E.S.K. Menon and A.G. Fox, Detection, Distribution and Quantification of Carbon in Steel Microstructures by PEELS, Microsc. Microanal., 2002, 8, p 392-402

19. C.P. Scott and J. Drillet, A Study of Carbon Distribution in Retained Austenite, Scr. Mater., 2007, 56, p 489-492

20. I.B. Timokhina, H. Beladi, X.Y. Xiong, Y. Adachi, and P.D. Hodgson, Nanoscale Microstructure Characterization of a Nanobainitic Steel, Acta Mater, 2011, 59, p 5511-5522

21. C. Garcia-Mateo, F.G. Caballero, M.K. Miller, and J.A. Jimenez, On Measurement of Carbon Content in Retained Austenite in a Nanostructured Bainitic Steel, J. Mater. Sci., 2012, 57, p 1004-1010

22. Q. Luo, M. Kitchen, V. Patel, and S. Magowan, Carbon Partitioning and Structure Evolution in the Hardening Treatments of High Strength Steel, The 20th Congress of International Federation for Heat Treatment and Surface Engineering, 2012, p 111-117

23. G.V. Kurdjumov, Martensitic Crystal-Lattice, Mechanism of AusteniteMartensite Transformation and Behaviour of Carbon-Atoms in Martensite, Metall. Trans., 1976, 7A, p 999-1011

24. L. Dabrowski, J. Suwalski, B. Sidshimov, and V. Christov, Investigations of Ordering Dynamics in Carbon Martensite, Acta Metall. Mater, 1994, 42, p 2375-2380

25. A. Udyansky, A.J. von Pezold, V.N. Bugaev, M. Friak, and J. Neugebauer, Interplay Between Long-Range Elastic and Short-Range Chemical Interactions in Fe-C Martensite Formation, Phys. Rev. B, 2009, 79, p 224112

26. S. Matas and R.F. Hehemann, Retained Austenite and the Tempering of Martensite, Nature, 1960, 187, p 685-686

27. M. Sarikaya, G. Thomas, J.W. Steeds, S.J. Barnard, and G.D.W. Smith, Solute Element Partitioning and Austenite Stabilization in Steels, Proceedings of an International Conference on Solid to Solid Phase Transformations, H.I. Aaronson, Ed., TMS, Warrendale, 1982, p 14211425

28. D.F. Lahrman, R.D. Field, R. Darolia, and H.L. Fraser, Investigation of Techniques for Measuring Lattice Mismatch in a Rhenium Containing Nickel Base Superalloy, Acta Mater, 1988, 36, p 1309-1320 
29. S. Tian, M. Wang, H. Yu, X. Yu, T. Li, and B. Qian, Influence of Element Re on Lattice Misfits and Stress Rupture Properties of Single Crystal Nickel-Based Superalloys, Mater. Sci. Eng. A, 2010, 527, p 4458-4465

30. Q. Luo, K. Chi, S. Li, and P. Barnard, Microstructural Stability and Lattice Misfit Characterisations of Nimonic 263, Proceedings of the ASME 2012 Pressure Vessels \& Piping Division Conference (PVP2012), 2012, Vol. 6, Pts A \& B, p 197-206

31. A. Stormvinter, G. Miyamoto, T. Furuhara, P. Hedstrom, and A. Borgenstam, Effect of Carbon Content on Variant Pairing of Martensite in Fe-C Alloys, Acta Mater., 2012, 60, p 7265-7274

32. S. Morito, K. Oh-Ishi, K. Hono, and T. Ohba, Carbon Enrichment in Retained Austenite Films in Low Carbon Lath Martensite Steel, ISIJ Int., 2011, 51, p 1200-1202
33. Q. Luo, M. Kitchen, V. Patel, M. Filleul, and D. Owens, PartialIsothermally-Treated Low Alloy Ultrahigh Strength Steel with Martensitic/Bainitic Microstructure, Proceedings HSLA Steels 2015, Microalloying 2015 \& Offshore Engineering Steels 2015, The Chinese Society for Metals and Chinese Academy of Engineering, November 11-13, 2015, Hangzhou, China, TMS 2016, p 433-438

34. H. Kitahara, R. Ueji, N. Tsuji, and Y. Minamino, Crystallographic Features of Lath Martensite in Low-Carbon Steel, Acta Mater., 2006, 54, p 1279-1288

35. H. Beladi, Y. Adachi, I. Timokhina, and P.D. Hodgson, Crystallographic Analysis of Nanobainitic Steels, Acta Mater., 2009, 60, p 455-458

36. V.G. Gavriljuk, B.D. Shanina, and H. Berns, On the Correlation Between Electron Structure and Short Range Atomic Order in IronBased Alloys, Acta Mater., 2000, 48, p 3879-3893 